\title{
Obstetric Outcomes of Women Who Sustained Traumatic Spinal Injury during Pregnancy: A Systematic Review
}

\author{
Aatik Arsh, Haider Darain \\ KMU Institute of Physical Medicine and Rehabilitation, Khyber Medical University, Peshawar, Pakistan
}

\begin{abstract}
Literature regarding pregnant women who sustained traumatic spinal injury during pregnancy is limited. Existing papers are mainly case reports, and this indicates a need for more high-quality research evidence in this area. Therefore, this study was designed to systematically review available literature that reported demographic information, clinical characteristics, and obstetric outcomes of women who sustained spinal injury during pregnancy. Studies published from the earliest record to January 2019, which reported traumatic spinal injury during pregnancy, were included. Studies that have reported pregnancy in patients with pre-existing spinal injury, review articles, commentaries, letter to editors, conference papers, and studies published in languages other than English were excluded. Sixteen studies reporting on 26 women were included. Their mean age was $26.7 \pm 5.5$ years. Motor vehicle accidents were the main cause of spinal injury $(n=15,58 \%)$. Of the cases, four women sustained spinal injury during the 1st trimester of pregnancy, of which one had a spontaneous abortion, one delivered a baby with arthrogryposis multiplex congenital disorder, and the third one delivered a premature baby who died shortly after the delivery. Fifteen women sustained injury during their 2nd trimester, of which 14 delivered normal babies, while six sustained spinal injury during the 3rd trimester. Of these women, five delivered normal babies at term. Outcomes of pregnancy appear significantly affected if a spinal injury occurs during the 1st trimester of pregnancy; however, injuries sustained during the 2nd and 3rd trimesters can have good obstetric outcomes.
\end{abstract}

Keywords: Obstetric outcomes; Pregnancy; Spine; Trauma

\section{Introduction}

Traumatic spinal injuries during pregnancy are less frequent; however, these injuries are associated with inconceivable suffering and financial costs $[1,2]$. Trauma during pregnancy is one of the leading non-obstetric causes of fetal deaths [3]. In cases where the fetus survives, complications such as low birth weight and prematurity have been reported in the literature [4,5]. Besides fetal complica- tions, acute spinal injury in pregnant women brings a high risk of maternal morbidity and mortality [5-7]. Severe spinal injury can lead to spinal cord injury (SCI). In these patients, spinal injury and SCI-specific complications such as paralysis, bowel and bladder incontinence, deep venous thrombosis, hypotension, autonomic dysreflexia (AD), and vertebral instability present complex challenges to obstetricians, surgeons, and rehabilitation specialists [6-10]. Thus, an interdisciplinary team approach is neces-

Received Apr 26, 2020; Revised Dec 26, 2020; Accepted Jan 18, 2021

Corresponding author: Aatik Arsh

KMU Institute of Physical Medicine and Rehabilitation, Khyber Medical University, Peshawar, Pakistan

Tel: +92-3446997046, Fax: +92-919217266, E-mail: aatikarsh@kmu.edu.pk 
sary to manage this unique patient population. However, financial constraints are present in developing countries, and relevant facilities are not always available for this uncommon condition [7].

Spinal injury and SCI commonly occur in young individuals, and the majority of women who sustain these injuries are of childbearing age $[7,9,10]$. Some studies have investigated the outcomes of pregnancy and rehabilitation in women with pre-existing SCI; however, the literature regarding pregnant women who sustained spinal injury during pregnancy is limited [8]. Existing literature mainly consists of case reports, which indicates a need for more high-quality research evidence in this area. Therefore, this study was designed to systematically review available literature that reported demographic information, clinical characteristics, and obstetric outcomes of women who sustained spinal injury during pregnancy. The research question was "What are the clinical features and obstetric outcomes of women who sustained traumatic spinal injury during pregnancy?"

\section{Methods}

A systematic review was conducted according to PRISMA (Preferred Reporting Items for Systematic Reviews and Meta-Analyses) guidelines from February to July 2019. The study was registered with PROSPERO (www.crd. york.ac.uk/PROSPERO) under the registration number CRD42018115815. A literature search was performed in March 2019 using databases of PubMed/MEDLINE, AMED, EMBASE, HMIC, BNI, PsycINFO, CINAHL (EBSCO), and Health Business Elite. The literature search was performed using the following terms: spinal cord (injury, damage, compression, ischemia, trauma, contusion, laceration, transection, syndrome), OR spinal (fracture, sublaxation, dislocation, injury, trauma), OR cervical vertebrae injuries, OR lumbar vertebrae injuries, OR thoracic vertebrae injuries, OR SCI, OR paraplegia, OR quadriplegia, OR tetraplegia, AND pregnancy, OR pregnant women, OR antenatal, OR prenatal, OR perinatal, OR gravid, OR gestation. Truncations were used when appropriate. Following search limits were applied in PubMed database: species (humans) and sex (female).

Search results were imported into the reference manager Endnote X7 (Clarivate, Philadelphia, PA, USA) to remove duplicates. After duplicate removal, two authors (A.A. and H.D.) independently screened the titles and abstracts of the studies and divided the articles into "relevant" and "irrelevant" categories according to the inclusion and exclusion criteria. All discrepancies were resolved by consensus meeting/discussion, and the principal author (A.A.) made the final decision. Research articles published from the earliest record to January 2019 that reported traumatic spinal injury during pregnancy were included. Studies on non-traumatic spinal injury, such as due to osteoporosis or neoplastic causes, were excluded. Only peer-reviewed articles in English language were included. Reference list of articles were systematically scanned to find additional related studies. Reviews, commentaries, letter to editors, and conference papers were excluded.

The principal author (A.A.) studied the full texts of the articles selected, and the following data were extracted from each included study: principal author, year of publication, country, patient age, cause of injury, gestational age at the time of injury, radiological findings, neurological deficits, spine management (surgical/conservative), obstetric outcomes, complications, and follow-up status. Extracted data were checked by the second author (H.D.), and discrepancies were resolved by consensus. The methodological quality of the included studies was evaluated by adapting the methods of Bazerbachi et al. [11]. Given that there were no available validated tools to assess the methodological quality of case reports and case series, Bazerbachi et al. [11] derived items from the Newcastle-Ottawa Scale that were appropriate for non-comparative case reports and case series studies. They removed two items (comparability and adjustment) from the Newcastle-Ottawa Scale for their systematic review of non-comparative case reports and case series. According to Bazerbachi et al. [11], the quality of the report is good when all five criteria were fulfilled, moderate when four were fulfilled, and low when $\leq 3$ were fulfilled. The same scoring method was used in the present systematic review. IBM SPSS ver. 23.0 (IBM Corp., Armonk, NY, USA) was used to calculate the mean, simple frequency, percentages, and proportion from the total case reports.

\section{Results}

The initial search identified 461 research articles. One study was identified through additional sources [12]. Following removal of duplicates, 399 studies were left, of which 19 were excluded because they were published 

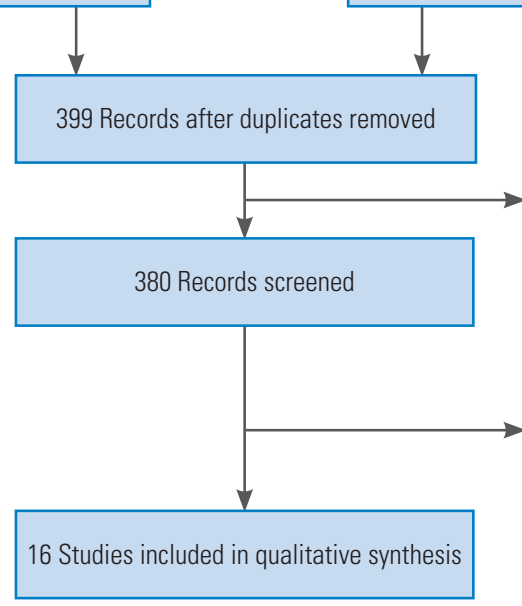

19 Non-English articles removed

- Non-traumatic spinal injury

364 Records excluded

- Pregnancy in women with pre-existing SCI

- Minor spinal injuries without associated vertebrae fracture or SCI

- Reviews/expert opinions

Fig. 1. Study selection flowchart. SCI, spinal cord injury.

in languages other than English. Titles and abstracts of the remaining 380 articles were screened, and $361 \mathrm{ex}-$ cluded because either they reported spinal injury due to non-traumatic causes or pregnancy in women with pre-existing SCI or they reported minor spinal injuries without associated vertebral fracture or SCI. Of the remaining 19 studies, three studies [13-15], which were narrative reviews or expert opinions, were excluded (Fig. 1). Another study reported two cases, of which one was of non-traumatic origin [9]. The non-traumatic case was removed, leaving the study solely with the traumatic case. Finally, 16 publications reporting on 26 cases were included in the systematic review. Eleven (68.8\%) of the included studies had poor methodological quality, while the remaining 5 (31.2\%) had moderate methodological quality $[2,6,7,9,10,12,16-25]$ (Table 1 ).

The studies included were published from 2000 to 2018. Geographical distribution of these studies is as follows: Bulgaria $(n=2)$, Turkey $(n=1)$, South Africa $(n=1)$, Nigeria $(\mathrm{n}=3)$, United States $(\mathrm{n}=1)$, Germany $(\mathrm{n}=1)$, Brazil $(\mathrm{n}=1)$, Australia $(n=1)$, Croatia $(n=1)$, India $(n=3)$, Pakistan $(\mathrm{n}=5)$, Saudi Arabia $(\mathrm{n}=1)$, and Yemen $(\mathrm{n}=5)$.

The subjects' age ranged from 19 to 39 years, with a mean age of $26.7 \pm 5.5$ years. Motor vehicle accident (MVA) was the cause of injury $(n=15,58 \%)$, followed by falls $(n=8$, $31 \%)$ and gunshot wound (GSW) $(\mathrm{n}=3,11 \%)$. Less than half $(n=12,46 \%)$ of the cases had cervical injuries, while the remaining 14 (54\%) had thoracic or lumbar injuries. A large proportion $(n=17,65 \%)$ of patients were managed surgically, while the remaining patients $(n=9,35 \%)$ were treated conservatively. There were 4 (15\%), 15 (58\%), and $6(23 \%)$ women who sustained injuries during the 1 st, 2nd, and 3rd trimesters, respectively. In one case (4\%), the patient sustained injury in the 6th lunar month of her pregnancy.

Moreover, 20 women (77\%), of which 10 had cervical injuries and 10 had thoracic/lumbar injuries, delivered normal healthy babies. Of these women, 13 underwent spinal surgery and seven were managed conservatively. Obstetric outcomes were not satisfactory in four women who sustained injury during the 1st trimester; one had a spontaneous abortion, another delivered a baby with arthrogryposis multiplex congenital disorder, and the third one delivered a premature baby who died an hour after delivery. The outcome of the fourth women cannot be reported, as no information was provided on follow-up or delivery. Outcomes of women who sustained spinal injury during the 2nd trimester were satisfactory, and of the 15 women, 14 delivered healthy babies, while one delivered a premature baby. Similar satisfactory outcomes were reported for women who sustained spinal injury in the 3rd trimester of pregnancy; of the six women, five delivered normal babies, while one delivered at preterm. The latter woman developed $\mathrm{AD}$ during labor and died shortly after the delivery; however, her baby survived. The woman who sustained injury during the 6th lunar month of her pregnancy had a spontaneous abortion. 


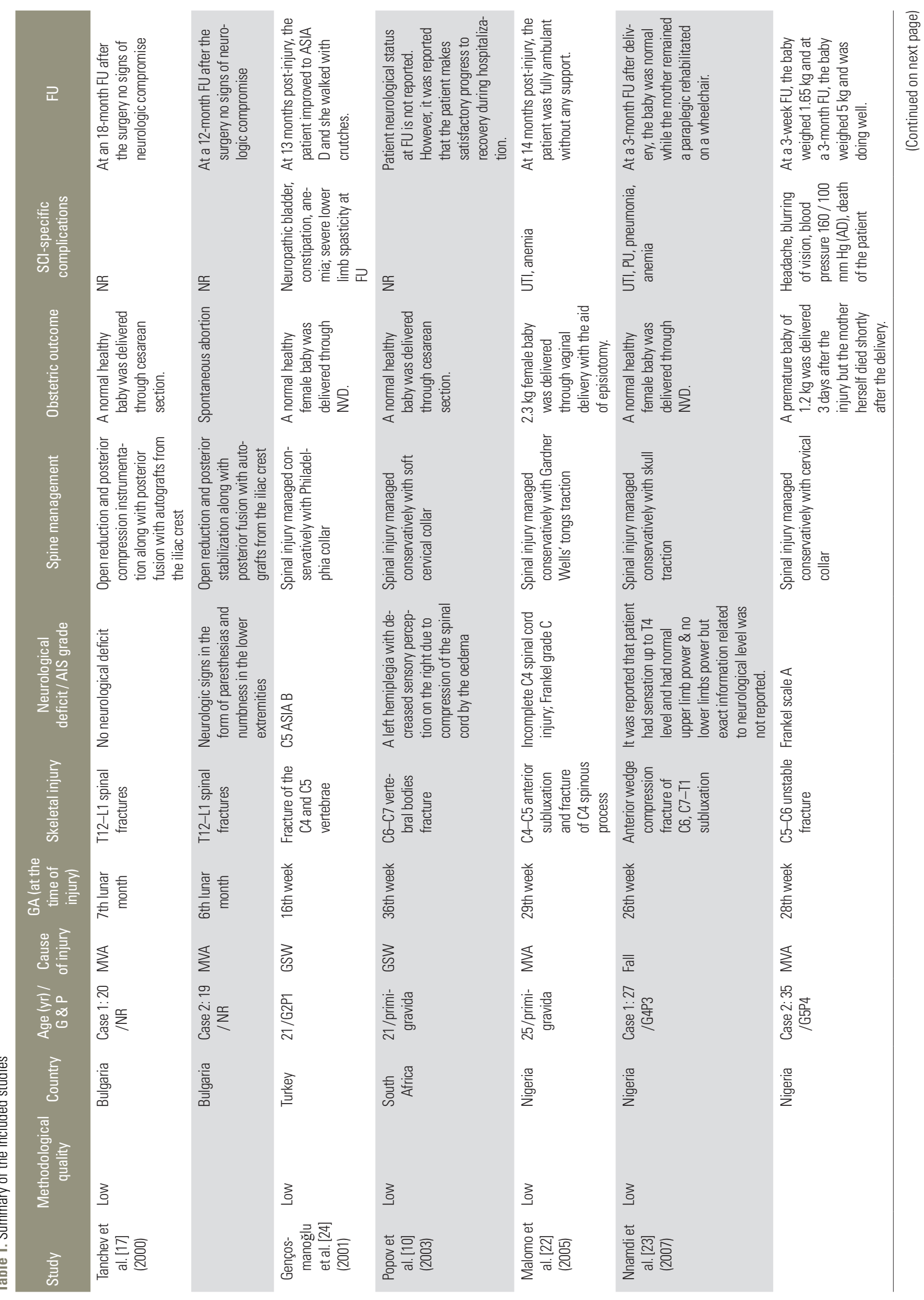



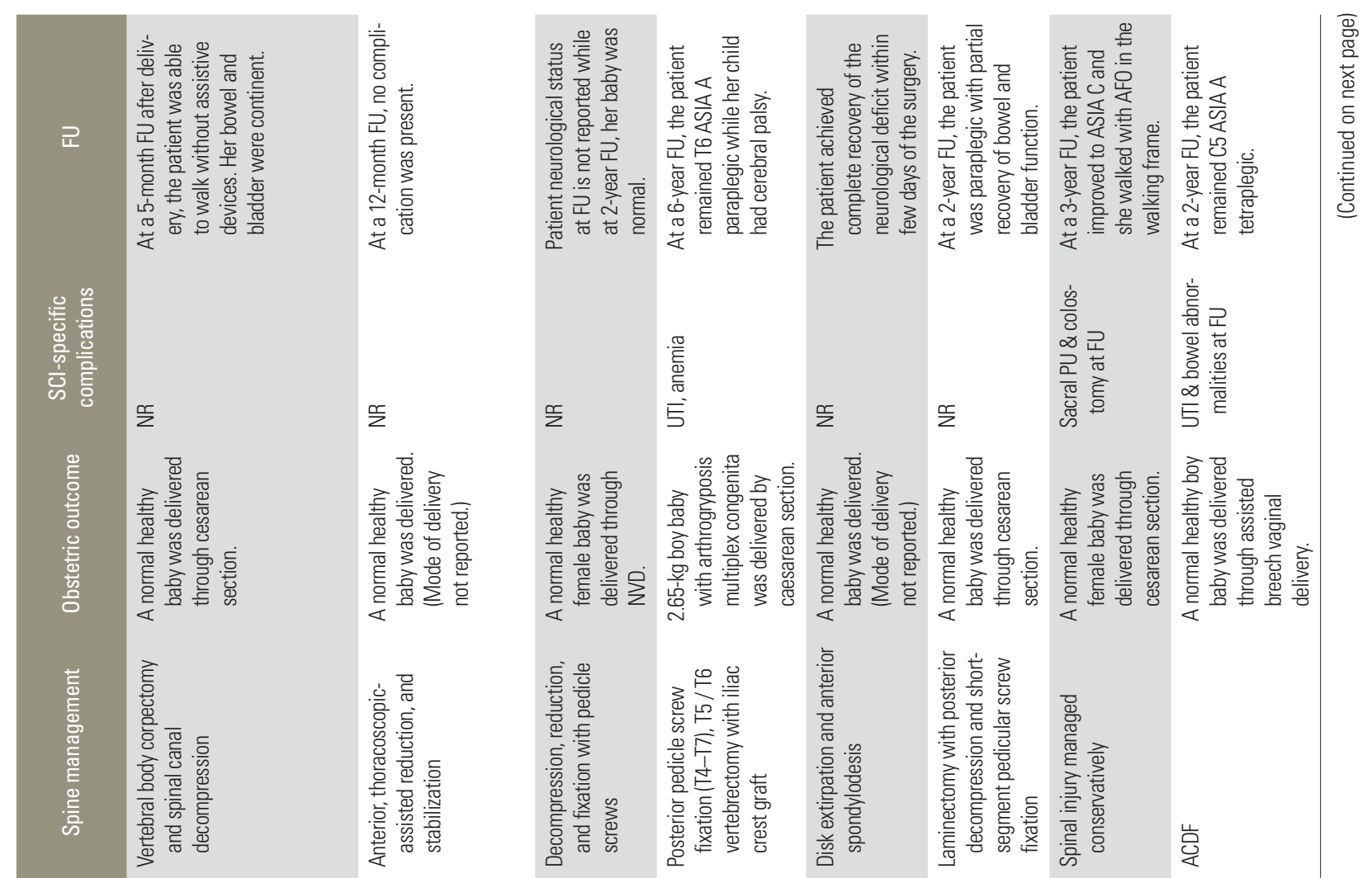

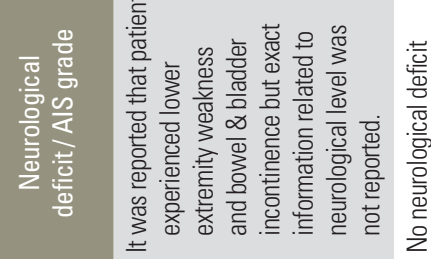
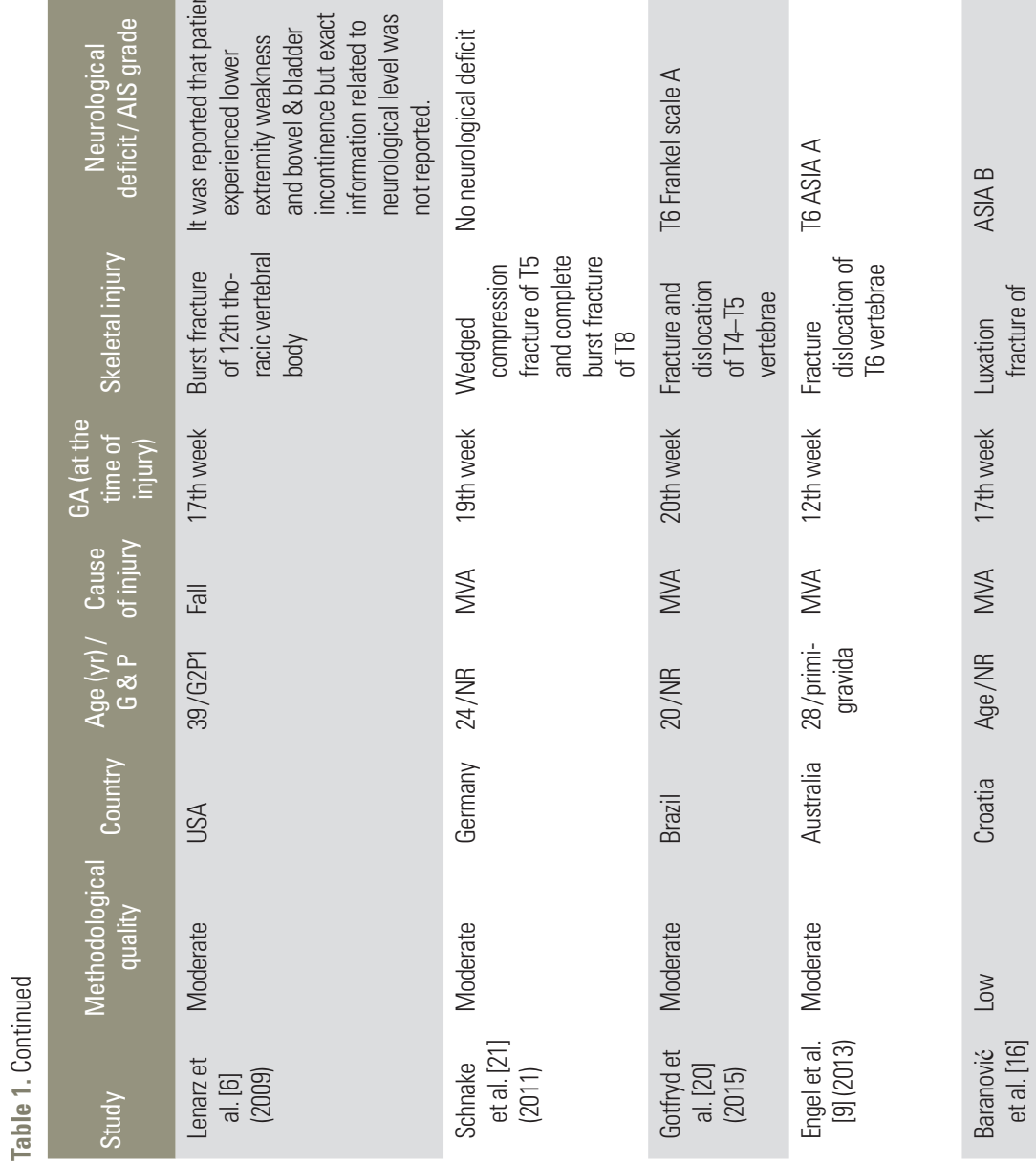

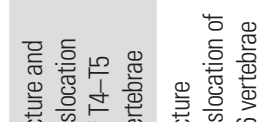
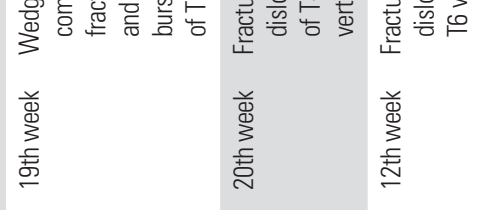

变

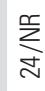

产

兽

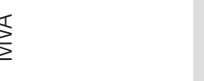

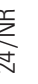

空

率

屁

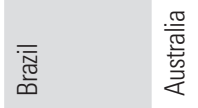

$\frac{\mathbb{2}}{\frac{\pi}{\pi}}$

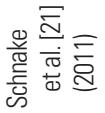

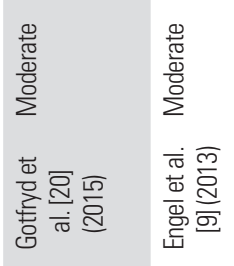

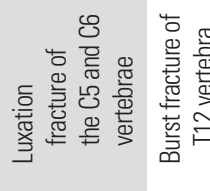

尊酸

率

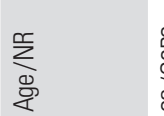

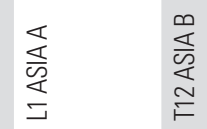

$\frac{\sqrt{3}}{\frac{1}{3}}$
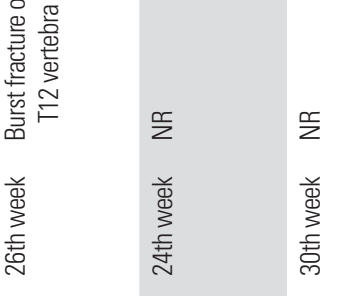

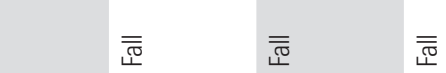

爰

葛

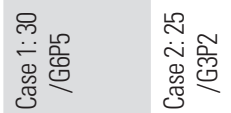

宽

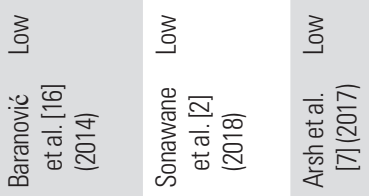




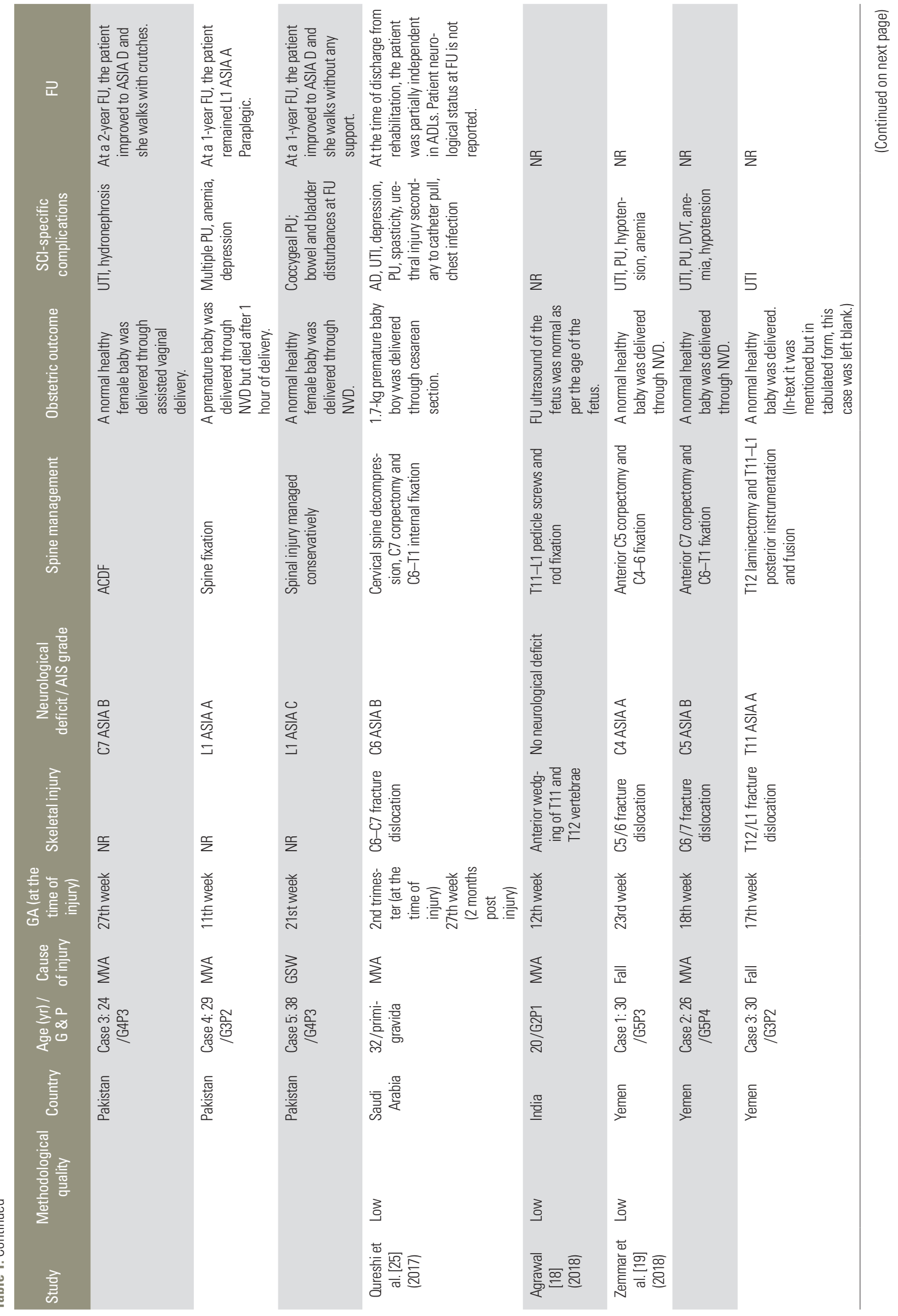




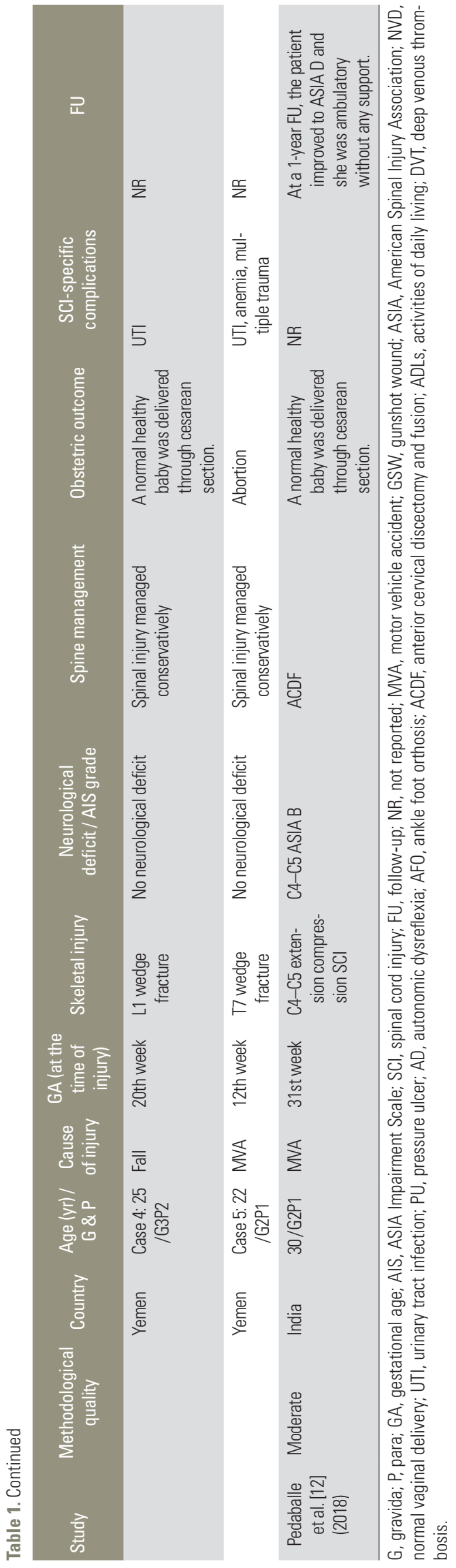

\section{Discussion}

Spinal injuries lead to an array of physical suffering [16-18]. Moreover, these injuries have profound social, financial, and psychological implications $[18,26]$. Consequences of spinal injuries are significantly exacerbated in women who sustained spinal injuries during pregnancy, and these effects can be worsened further when clinicians are uncertain on the best procedures to save or treat the patient and her baby. Frequent and effective communication among health care professionals, including obstetricians, surgeons, and rehabilitation specialists who form a part of an interdisciplinary team, is necessary for the best management of these patients $[7,9,12]$. Traumatic spinal injuries during pregnancy are not very common; therefore, limited information exists about the clinical characteristics and obstetric outcomes in this population $[19,20]$. This systematic review aimed to assimilate data from all quality, peer-reviewed research to document the current knowledge base pertaining to demographics, clinical characteristics, and obstetric outcomes of women who sustained spinal injury during their pregnancies.

Fall from height, MVA, and GSW are the leading causes of traumatic spinal injuries in both developed and developing countries [27,28]. Our findings indicate that MVA was the most common cause of spinal injury in pregnant women. This aligns with a previous study reporting that MVA is the leading cause of trauma during pregnancy and accounts for approximately $82 \%$ of fetal deaths during trauma [29]. Most of the patients in this review sustained thoracic or lumbar injuries, which are commonly injured areas of the spine during trauma [30,31]. A small proportion of the included cases had injury during the 1st trimester, while the majority had spinal injury during the 2nd and 3rd trimesters. This finding agrees with the results of previous studies that maternal injuries during the 1 st trimester of pregnancy are uncommon [32,33].

About two-thirds ( $n=17,65 \%)$ of patients in this review received surgical treatment. While surgical management of spinal fracture in pregnancy carries a risk to both the mother and fetus due to positioning, blood loss, and inherent risk of anesthesia, pregnancy is not a contraindication for surgical management of the spine $[2,17,21]$. Numerous studies have suggested that conservative or surgical management of the spine does not affect pregnancy outcomes in women who sustained spinal injury during pregnancy $[2,7,19,22-24]$. Some studies have re- 
ported that maternal surgery during pregnancy does not increase the risk of fetal congenital anomalies, though evidence suggests that surgery during pregnancy increases the risk of preterm delivery. Patients with unstable spine require surgical treatment, with the objective of achieving mechanical stabilization of the spine $[7,20,25,34]$. Utilizing the prone position for spinal surgery is feasible during the early stages of pregnancy but is contraindicated after 12 weeks of gestation [19,34]. The lateral decubitus position is recommended in the later stages of pregnancy because the risk of aortocaval compression is higher in the supine position $[25,26,34]$. In this systematic review, drawing conclusion about positioning for surgery during pregnancy from the studies included was not possible because of the high heterogeneity in the surgical procedures and majority of the studies did not report positioning of patients during surgery. According to Sonawane et al. [2], it is prudent to postpone the surgery until after delivery if the patient is near term; however, earlier weeks of pregnancy, unstable spine, and incomplete neurological deficit are clear-cut indications for surgery. Of the cases included in this review, nine were managed conservatively. Conservative management mainly reduces the risk of premature birth and prevents the fetus from being exposed to radiations [12]. However, conservative management increases the risk of developing SCI-specific secondary complications such as pressure ulcers and deep venous thrombosis. Moreover, delay in starting aggressive rehabilitation can lead to psychological issues $[7,12,22]$. In this review, the three women who sustained spinal injury due to GSW were managed conservatively; because spinal instability due to GSW rarely occurs, most GSW cases are managed conservatively [24]. In clinical practice, patients with spinal injury whose spine is either stable or closed reduction is possible are managed conservatively $[7,22,23]$. However, presenting recommendations regarding conservative or surgical management during pregnancy may be beyond the scope of this review. To simplify, the nature of the spinal injury, stage of pregnancy, neurological status, and preferences of the patients are certain factors that should be considered before treatment decision $[12,22,35]$.

Anesthesia for the management of spinal injury during pregnancy is rarely described in literature. Physiological changes that accompany pregnancy can affect maternal and fetal safety during anesthesia $[16,36]$. Changes in the cardiorespiratory system during pregnancy should be considered when conducting anesthesia for pregnant women with spinal injury. Because oxygen reserve is generally reduced in pregnant women and anesthesia further decreases functional residual capacity, even short periods of apnea can have devastating results. Therefore, close monitoring of maternal oxygenation throughout the procedure is recommended [37,38]. Another major problem is that pregnant women are at risk of anesthetic overdose. Generally, all anesthetic agents have the potential to cross the placenta; nevertheless, none of the commonly used anesthetic agents have teratogenic effects. Despite this, fetal hypoxia resulting from anesthesia can be dangerous. Thus, anesthesiologists generally recommend postponing surgery until the 1st trimester. From a logical point of view, fetal exposure to anesthetic agents will be less during spinal anesthesia in contrast to general anesthesia; nonetheless, very few studies have supported the fact that spinal anesthesia is safer than general anesthesia and vice versa [37]. Of the total 16 studies included in this review, only three discussed anesthetic considerations for spinal surgery during pregnancy $[12,16,25]$ and used general anesthesia. None of these studies reported any anesthesiaspecific complication in patients who received anesthesia for the management of spinal injury during pregnancy. The lack of anesthesia-specific information in the included studies is one of the major limitations of this systematic review.

Rehabilitation of pregnant women with acute spinal injury is challenging due to difficulties with positioning, nursing care, mobilization, and transfers [25]. Increased weight and protuberant abdomen pose additional difficulties in transfers and mobilization, leading to greater dependence. Fear of fetal loss due to trauma, misconceptions about pregnancy, disability, and rehabilitation are some other factors that can delay rehabilitation in these patients [7]. No specific guidelines are available for the rehabilitation of this patient population, which is another obstacle to their rehabilitation. Pregnant women with acute spinal injury or SCI can be rehabilitated in the same way as other patients with spinal injury or SCI as long as precautions are taken to account for the growing fetus, and special attention should be given to changing activities according to gestational age. In addition to normal physiological changes of pregnancy, patients with acute spinal injury undergo physiological changes caused by their injury, which increases the risk of developing secondary complications [19]. For example, pregnant women at baseline are five times more likely to develop deep venous 
thrombosis than non-pregnant women of the same age, while SCI further increases the risk due to immobilization $[2,25]$. Besides deep venous thrombosis, other complications reported in this review were urinary tract infections, pressure sores, anemia, depression, and spasticity. $\mathrm{AD}$ is another major and life-threatening complication in pregnant patients with SCI and injury level at or above T6. It can occur at any stage, but is common during labor and delivery $[7,23,25]$. In this review, two women developed $\mathrm{AD}$ : one woman developed $\mathrm{AD}$ during delivery and died shortly after delivering a premature baby, and the other woman had three episodes of AD during cervical spine fixation.

A few studies have reported that severe trauma during pregnancy has negative outcomes in most cases $[3,5,9]$. Trauma has been associated with an increased risk of spontaneous abortion, premature labor, fetal distress, and stillbirth [39]. However, the results of our systematic review revealed that $77 \%$ of the women who sustained injury during pregnancy delivered normal healthy babies. The majority of the included studies have reported that an interdisciplinary team approach is the only way to manage this unique patient population $[2,6,7,12]$. Our review indicates that evidence is insufficient to suggest that spinal injury during pregnancy is strongly associated with fetal loss and abnormality. However, 1st trimester spinal injury can result in a myriad of fetus-related complications $[3,9,10,29]$, and our findings also indicate poor obstetric outcomes in women who sustained spinal injury during the 1st trimester. The exact mechanisms on how traumatic spinal injury affects obstetric outcomes are not fully understood; however, placental abruption or uterine rupture due to trauma, spinal shock, direct fetal injuries, vertebral dislocations, $\mathrm{AD}$, and other trauma-specific complications can be the cause of poor obstetric outcomes in women who sustain traumatic spinal injury during pregnancy. The authors failed to find any literature that explains whether injuries to the spinal cord or damage to neural signaling pathways have direct association with obstetric outcomes. Given the scarcity of high-quality research studies, the results of this review do not provide high-quality evidence. According to Ackley et al. [40], evidence from systematic reviews of descriptive studies provide level $\mathrm{V}$ evidence [41]; therefore, this study provides poor-quality evidence.

\section{Conclusions}

To our knowledge, this systematic review is the first to report obstetric outcomes in women who sustained traumatic spinal injury during pregnancy. The results of this review suggest that pregnancy outcomes are significantly affected if injury occurs during the 1st trimester of pregnancy; however, injuries sustained during the 2nd and 3 rd trimesters can have good obstetric outcomes. Given the limited available literature, we are unable to draw conclusions about what constitutes optimal care and management of these patients, but it is likely to include an interdisciplinary team approach, especially given the challenges of managing both the patient and the growing fetus. Future research should focus on the development of clinical guidelines for the management of these patients. Moreover, worldwide data on these patients should be collected to truly determine the influence of spinal injury on obstetric outcomes.

\section{Conflict of Interest}

No potential conflict of interest relevant to this article was reported.

\section{Author Contributions}

AA was responsible for designing the review protocol, conducting the search, screening potentially eligible studies, extracting and analyzing data, interpreting results, creating 'summary of findings' tables, and writing the report. $\mathrm{HD}$ was responsible for designing the review protocol and screening potentially eligible studies. He also contributed to data extraction and provided feedback on the report.

\section{References}

1. Kuczkowski KM. Labor analgesia for the parturient with spinal cord injury: what does an obstetrician need to know? Arch Gynecol Obstet 2006;274:10812.

2. Sonawane DV, Nemade PS, Chandanwale A, Pawar E, Jagtap SA. Management of fracture dorso-lumbar spine in a pregnant female by surgery in prone position. Eur Spine J 2018;27(Suppl 3):270-5.

3. Weiss HB, Songer TJ, Fabio A. Fetal deaths related to maternal injury. JAMA 2001;286:1863-8. 
4. Sperry JL, Casey BM, McIntire DD, Minei JP, Gentilello LM, Shafi S. Long-term fetal outcomes in pregnant trauma patients. Am J Surg 2006;192:715-21.

5. Brown HL. Trauma in pregnancy. Obstet Gynecol 2009;114:147-60.

6. Lenarz CJ, Wittgen CM, Place HM. Management of a pregnant patient with a burst fracture causing neurologic injury: a case report. J Bone Joint Surg Am 2009;91:1747-9.

7. Arsh A, Darain H, Ilyas SM, Zeb A. Consequences of traumatic spinal cord injury during pregnancy in Pakistan. Spinal Cord Ser Cases 2017;3:17041.

8. Ghidini A, Healey A, Andreani M, Simonson MR. Pregnancy and women with spinal cord injuries. Acta Obstet Gynecol Scand 2008;87:1006-10.

9. Engel S, Ferrara G. Obstetric outcomes in women who sustained a spinal cord injury during pregnancy. Spinal Cord 2013;51:170-1.

10. Popov I, Ngambu F, Mantel G, Rout C, Moodley J. Acute spinal cord injury in pregnancy: an illustrative case and literature review. J Obstet Gynaecol 2003;23:596-8.

11. Bazerbachi F, Haffar S, Sugihara T, et al. Peribiliary cysts: a systematic review and proposal of a classification framework. BMJ Open Gastroenterol 2018;5:e000204.

12. Pedaballe AR, Chhabra HS, Tandon V, Chauhan P, Verma R. Acute traumatic cervical spinal cord injury in a third-trimester pregnant female with good maternal and fetal outcome: a case report and literature review. Spinal Cord Ser Cases 2018;4:93.

13. Kuczkowski KM, Fouhy SA, Greenberg M, Benumof JL. Trauma in pregnancy: anaesthetic management of the pregnant trauma victim with unstable cervical spine. Anaesthesia 2003;58:822.

14. Dawood R, Altanis E, Ribes-Pastor P, Ashworth F. Pregnancy and spinal cord injury. Obstet Gynaecol 2014;16:99-107.

15. Chilkoti GT, Mohta M, Duggal S, Saxena AK. Anaesthetic concerns of a pregnant patient with Pott's spine for spine surgery in prone position. Indian J Anaesth 2016;60:518-9.

16. Baranovic S, Maldini B, Cengic T, Kolundzic R. Anesthetic management of acute cervical spinal cord injury in pregnancy. Acta Clin Croat 2014;53:98-101.

17. Tanchev P, Dikov D, Novkov H. Thoracolumbar distraction fractures in advanced pregnancy: a contribu- tion of two case reports. Eur Spine J 2000;9:167-70.

18. Agrawal A. Management of a case of spinal cord injury sustained during pregnancy. J Spinal Surg 2018;5:132-4.

19. Zemmar A, Al-Jradi A, Ye V, et al. Medical and surgical management of acute spinal injury during pregnancy: a case series in a third-world country. Surg Neurol Int 2018;9:258.

20. Gotfryd AO, Franzin FJ, Poletto PR, Neto NJ, Junior RC, Junior LC. Fracture-dislocation of the thoracic spine during second trimester of pregnancy: case report and literature review. Rev Bras Ortop 2015;47:521-5.

21. Schnake KJ, Scholz M, Marx A, Hoffmann R, Kandziora F. Anterior, thoracoscopic-assisted reduction and stabilization of a thoracic burst fracture (T8) in a pregnant woman. Eur Spine J 2011;20:1217-21.

22. Malomo AO, Emejulu JC, Odukogbe AA, Shokunnbi WA, Shokunbi MT. Conservative management of third trimester cervical spinal cord injury using Gardner-Wells tongs traction. Niger J Clin Pract 2005;8:46-50.

23. Nnamdi OS, Cajetan N. Traumatic spinal cord injury during pregnancy: report of two cases. J Obstet Gynecol India 2007;57:167-8.

24. Gencosmanoglu BE, Hanci M, Yucesoy G, Madazli R, Yilmaz H, Ozgen M. Spinal cord injury caused by gunshot wound during pregnancy. J Spinal Cord Med 2001;24:123-6.

25. Qureshi AZ, Ullah S, AlSaleh AJ, Ullah R. Spinal cord injury during the second trimester of pregnancy. Spinal Cord Ser Cases 2017;3:17052.

26. Darain H, Arsh A, Zeb A, Ilyas SM, Muhammad D, Khan MN. Epidemiology, clinical features and consequences of spinal cord injury in children. J Coll Physicians Surg Pak 2018;28:532-5.

27. Wyndaele M, Wyndaele JJ. Incidence, prevalence and epidemiology of spinal cord injury: what learns a worldwide literature survey? Spinal Cord 2006;44:523-9.

28. Chen Y, Tang Y, Vogel LC, Devivo MJ. Causes of spinal cord injury. Top Spinal Cord Inj Rehabil 2013;19:1-8.

29. Mattox KL, Goetzl L. Trauma in pregnancy. Crit Care Med 2005;33(10 Suppl):S385-9.

30. Sipski ML, Jackson AB, Gomez-Marin O, Estores I, Stein A. Effects of gender on neurologic and func- 
tional recovery after spinal cord injury. Arch Phys Med Rehabil 2004;85:1826-36.

31. Lee BB, Cripps RA, Fitzharris M, Wing PC. The global map for traumatic spinal cord injury epidemiology: update 2011, global incidence rate. Spinal Cord 2014;52:110-6.

32. Harland KK, Saftlas AF, Yankowitz J, Peek-Asa C. Risk factors for maternal injuries in a populationbased sample of pregnant women. J Womens Health (Larchmt) 2014;23:1033-8.

33. Shah KH, Simons RK, Holbrook T, Fortlage D, Winchell RJ, Hoyt DB. Trauma in pregnancy: maternal and fetal outcomes. J Trauma 1998;45:83-6.

34. Han IH, Kuh SU, Kim JH, et al. Clinical approach and surgical strategy for spinal diseases in pregnant women: a report of ten cases. Spine (Phila Pa 1976) 2008;33:E614-9.

35. El Masri Y WS. Traumatic spinal injury and spinal cord injury: point for active physiological conservative management as compared to surgical management. Spinal Cord Ser Cases 2018;4:14.
36. Moon TS, Sappenfield J. Anesthetic management and challenges in the pregnant patient. Curr Anesthesiol Rep 2016;6:89-94.

37. Wong CA. Analgesia and anesthesia for labor and delivery. Glob Libr Women's Med [Internet]. 2009 [cited 2020 Apr 10]:GLOWM.10216. Available from: https://doi.org/10.3843/GLOWM.10216.

38. Okeagu CN, Anandi P, Gennuso S, et al. Clinical management of the pregnant patient undergoing non-obstetric surgery: review of guidelines. Best Pract Res Clin Anaesthesiol 2020;34:269-81.

39. El-Kady D, Gilbert WM, Anderson J, Danielsen B, Towner D, Smith LH. Trauma during pregnancy: an analysis of maternal and fetal outcomes in a large population. Am J Obstet Gynecol 2004;190:1661-8.

40. Ackley B, Ladwig G, Swan BA, Tucker S. Evidencebased nursing care guidelines: medical surgical interventions. St. Louis (MO): Mosby Elsevier; 2008.

41. Evidence based practice toolkit: levels of evidence [Internet]. Winona (MN): Winona State University; 2021 [cited 2020 Apr 10]. Available from: https://libguides.winona.edu/c.php?g=11614\&p=61584. 